\title{
Precision Medicine Based on CFTR Genotype for People with Cystic Fibrosis
}

\author{
Iram Haq ${ }^{1,2}$, Maryam Almulhem (D)', Simone Soars', David Poulton ${ }^{2,3}$, Malcolm Brodlie $\mathbb{D}^{1,2}$ \\ 'Translational and Clinical Research Institute, Faculty of Medical Sciences, Newcastle University, Newcastle upon Tyne, UK; ${ }^{2}$ Paediatric Respiratory \\ Medicine, Great North Children's Hospital, Newcastle Hospitals NHS Foundation Trust, Newcastle upon Tyne, UK; ${ }^{3}$ Paediatrics, Ninewells Hospital, \\ NHS Tayside, Dundee, UK
}

Correspondence: Malcolm Brodlie, Paediatric Respiratory Medicine, Level 3, Clinical Resource Building, Royal Victoria Infirmary, Newcastle upon Tyne, NEI 4LP, UK, Tel +44 19I 2336I6I, Email malcolm.brodlie@ncl.ac.uk

\begin{abstract}
Cystic fibrosis (CF) is an autosomal recessive genetic condition that is caused by variants in the cystic fibrosis transmembrane conductance regulator gene. This causes multisystem disease due to dysfunction of the cystic fibrosis transmembrane conductance regulator (CFTR) ion channel at the apical surface of epithelia. Until recently, treatment was directed at managing the downstream effects in affected organs, principally improving airway clearance and treating infection in the lungs and improving malabsorption in the gastrointestinal tract. Care delivered by multidisciplinary teams has yielded incremental improvements in outcomes. However, the development of small-molecule CFTR modulator drugs over the last decade has heralded a new era of CF therapeutics. Modulators target the underlying defect and improve CFTR function. Either monotherapy or a combination of modulators is used depending on the specific genotype and class of CFTR disease-causing variants that an individual has. Both ivacaftor and the ivacaftor/tezacaftor/elexacaftor combination have been demonstrated to be associated with clinically very significant benefits in randomised trials and have rapidly been made available as part of standard care in many countries. CFTR modulators represent one of the best examples of precision medicine to date. They are expensive, however, and equity of access to them worldwide remains an issue. Studies and approvals are also ongoing for children under the age of 6 years for ivacaftor/tezacaftor/elexacaftor. Furthermore, no modulators are available for around $10 \%$ of the people with CF. In this review, we firstly summarise the genetics, pathophysiology and clinical problems associated with CF. We then discuss the development of CFTR modulators and key clinical trials to support their use along with other potential future therapeutic approaches.
\end{abstract}

Keywords: CFTR, ivacaftor, modulator, elexacaftor, tezacaftor

\section{Introduction}

Cystic fibrosis (CF) is an autosomal recessive disease that is caused by variants in the cystic fibrosis transmembrane conductance regulator (CFTR) gene. ${ }^{1,2}$ It is a condition involving the lungs, sweat glands, gastrointestinal and reproductive tracts among other systems. Traditionally, treatments in CF have involved the multidisciplinary management of downstream effects of CFTR dysfunction. This has included regular chest physiotherapy and antibiotics to limit the progression of bronchiectasis, pancreatic enzyme replacement therapy (PERT) to prevent malabsorption and optimise nutrition, and screening for and treatment of CF-related diabetes (CFRD) and liver disease (CFLD). ${ }^{2}$ Identification of patients with CF via newborn screening has facilitated early intervention and implementation of these therapies in many countries. ${ }^{3}$ Despite these efforts, the median age of survival for people with CF was around 40 years in $2010 .{ }^{4}$ The main cause of morbidity and mortality being chronic and progressive respiratory disease requiring lung transplantation in eligible patients. ${ }^{5}$

The recent development and availability of 'CFTR modulators' has been a major breakthrough for people with CF. These therapies function by targeting the underlying defect in the CFTR protein and a previously life-limiting disease may have a significantly improved outlook in the future.

In this review, we briefly outline the pathophysiology of CF then describe and discuss the development of CFTR modulators along with other strategies that hold promise for the future of CF therapeutics. 


\section{Pathophysiology of CF}

\section{The CFTR Gene and Protein}

The CFTR gene is located on the long arm at position q31-32 of chromosome $7 .{ }^{6}$ It encodes the CFTR protein, which consists of 1480 amino acids. CFTR is expressed at the apical surface of secretory epithelia including the sweat duct, respiratory system, pancreas and reproductive system. CFTR is a unique member of the ATP-binding cassette protein family in that it functions as an ion channel, transporting chloride and bicarbonate across the apical cell surface. ${ }^{7}$ CFTR is comprised of two homologous units, each containing a membrane-spanning domain (MSD 1 and MSD2), which form the ion channel pore across the plasma membrane and these are each connected to a cytoplasmic nucleotide-binding domain (NBD1 and NBD2). The homologous units are linked by a regulatory (R) domain (Figure 1). R domain phosphorylation, NBD-ATP binding and dimerization and subsequent MSD conformational change lead to CFTR activation, pore opening and gating of chloride secretion. ${ }^{8,9}$

\section{CFTR Variants}

Over 2000 CFTR variants have been described (Cystic Fibrosis Mutation Database http://www.genet.sickkids.on.ca). Only a relatively small proportion of these are disease-causing and these pathogenic variants (or mutations) have been classified according to their cellular and functional consequences (Table 1 and Figure 2). Class I variants arise from nonsense, frameshift or mRNA splicing defects and lead to either no protein synthesis or translation of a truncated protein. ${ }^{10}$ In class II variants dysfunctional protein processing causes an abnormally folded CFTR protein that cells mostly degrade with very little or no functional protein reaching the apical membrane. In class III variants although CFTR reaches the cell surface, channel gating is impaired. Class IV variants are principally conductance defects where CFTR is able to open and close, but chloride transport is reduced. ${ }^{11}$ Class V variants reduce the quantity of CFTR produced at the cell surface due to transcriptional regulation limitation. ${ }^{12}$ Class VI variants affect CFTR stability at the cell surface and result in increased protein turnover. ${ }^{13}$

This classification has provided a useful way to understand associated degrees of disease severity and response to different CFTR modulator therapies. Although many variants fall into specific classes, this is not always the case.

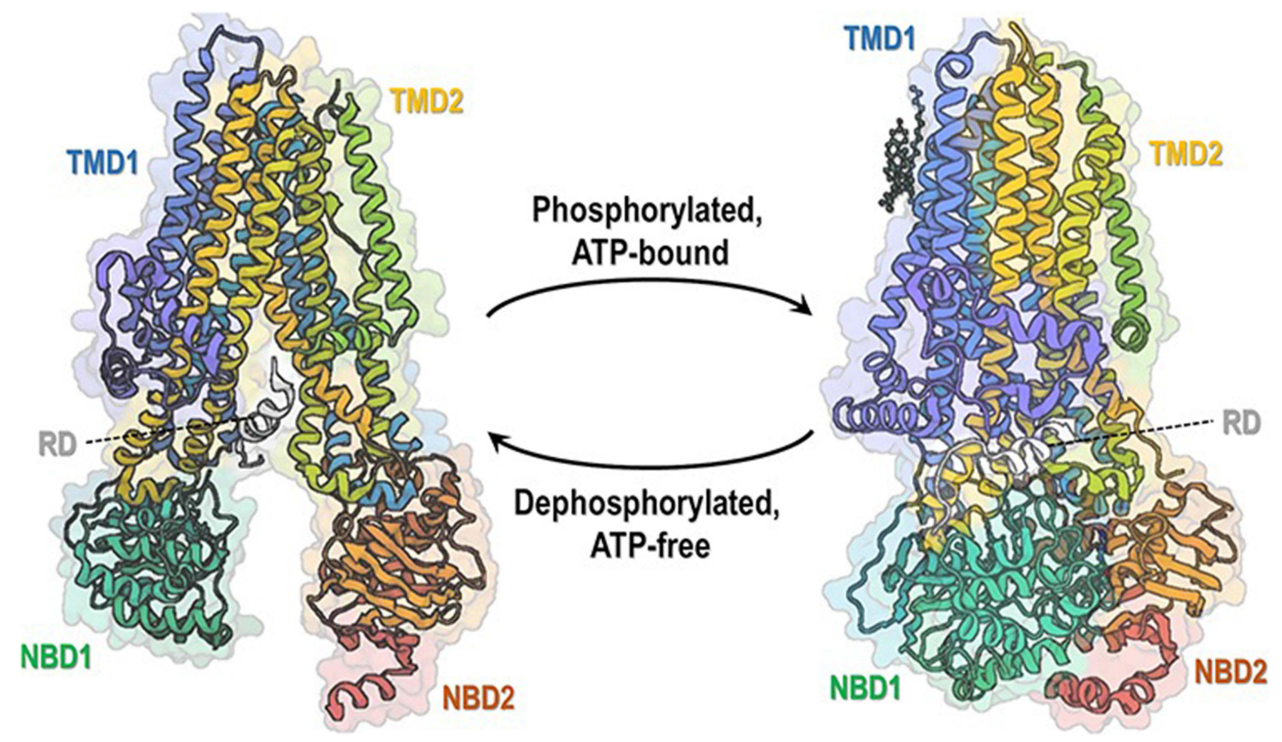

Figure I Current understanding of CFTR structure. Reprinted from Pinto MC, Silva IAL, Figueira MF, Amaral MD, Lopes-Pacheco M. Pharmacological Modulation of lon Channels for the Treatment of Cystic Fibrosis. J Exp Pharmacol. 2021;13:693-723. Copyright ( 202I Pinto et al. This work is published and licensed by Dove Medical Press Limited. ${ }^{99}$ CFTR structure is composed of five functional domains: two transmembrane domains (TMDI and TMD2), two nucleotide-binding domains (NBDI and NBD2) and an intrinsically disordered regulatory domain (RD). Ribbon diagram of two conformations of human CFTR: dephosphorylation, ATP-free conformation (left, PDB: 5UAK) (data from Liu et al 2017) ${ }^{100}$ and phosphorylated, ATP-bound conformation (right, PDB: 6MSM) (data from Zhang et al 2018). ${ }^{101}$ Notably, only a small portion of RD is depicted as most of its structure remains undetermined due to being intrinsically unstructured. 
Table I Classes of CFTR Disease-Causing Variants

\begin{tabular}{|c|c|c|c|c|c|c|}
\hline & Class I & Class II & Class III & Class IV & Class V & Class VI \\
\hline $\begin{array}{l}\text { Description of } \\
\text { defect }\end{array}$ & $\begin{array}{l}\text { Protein } \\
\text { synthesis }\end{array}$ & Protein trafficking & $\begin{array}{l}\text { Channel } \\
\text { gating }\end{array}$ & $\begin{array}{l}\text { Channel } \\
\text { conductance }\end{array}$ & $\begin{array}{l}\text { Reduced protein } \\
\text { synthesis }\end{array}$ & $\begin{array}{l}\text { Plasma membrane CFTR } \\
\text { stability }\end{array}$ \\
\hline Examples & $\begin{array}{l}\text { G542X } \\
\text { WI282X } \\
\text { R553X }\end{array}$ & $\begin{array}{l}\text { F508del } \\
\text { NI303K } \\
\text { I507del }\end{array}$ & $\begin{array}{l}\text { G55ID } \\
\text { S549R } \\
\text { GI349D }\end{array}$ & $\begin{array}{l}\text { RII7H } \\
\text { DII52H } \\
\text { R347P }\end{array}$ & $\begin{array}{l}3272-26 \mathrm{~A} \rightarrow \mathrm{G}, \\
3849+10 \mathrm{~kg} \mathrm{C} \rightarrow T\end{array}$ & $\begin{array}{l}\text { c. I20dell } 23 \\
\text { rPhe580del }\end{array}$ \\
\hline $\begin{array}{l}\text { Therapeutic } \\
\text { approach }\end{array}$ & $\begin{array}{l}\text { Genetic } \\
\text { therapies } \\
\text { Read-through } \\
\text { agents }\end{array}$ & $\begin{array}{l}\text { Corrector (and } \\
\text { potentiator) }\end{array}$ & Potentiator & Potentiator & Amplifier & Stabiliser \\
\hline Available drugs & - & $\begin{array}{l}\text { Elexacaftor/ tezacaftor/ } \\
\text { Ivacaftor } \\
\text { Lumacaftor/ Ivacaftor } \\
\text { Tezacaftor/ ivacaftor }\end{array}$ & Ivacaftor & Ivacaftor & - & - \\
\hline
\end{tabular}

F508del, for example, is the most common CFTR variant, arising from a deletion of phenylalanine at position 508. As a result, CFTR is misfolded (class II) but gating (class III) and stability (class VI) defects are also present. ${ }^{2}$

\section{Clinical Features, Diagnosis and Management of CF}

As mentioned already, several organs are affected in CF. The sweat glands and ducts function abnormally and in addition to genetic testing, measurement of sweat chloride is an important clinical test of CFTR function. Normally, the sweat gland secretes salt and water, which is then reabsorbed by the sweat duct. In CF, the sweat duct fails to absorb chloride, leading to a rise in salt concentration in the sweat. ${ }^{14}$

The lungs are severely affected in CF and progressive respiratory disease causes significant morbidity and indeed mortality. In the healthy airway, epithelia have a thin layer of liquid at the apical surface, known as the airway surface liquid (ASL). CFTR-mediated ion transport maintains ASL hydration, which is essential for physiological mucociliary clearance. In CF, dysfunctional ion transport disrupts ASL homeostasis. ${ }^{15}$ The viscoelastic properties of airway mucus are abnormal with several hypotheses proposed to explain this including the effects of ASL dehydration, reduced bicarbonate secretion leading to an acidic airway environment and impaired innate defense mechanisms against bacterial infection. ${ }^{2,7,15-17}$ This leads to a build-up of viscous secretions, mucus obstruction and chronic infection and inflammation. ${ }^{18}$ Staphylococcus aureus and Haemophilus influenzae are common pathogens detected in the lungs of children with $\mathrm{CF}$ in the first decade of life, while in older children Pseudomonas aeruginosa dominates with increasing rates of colonisation by early adulthood. ${ }^{19}$ Antibiotics are used to target specific pathogens as a strategy to minimise infection. Mucus clearance is improved with mucolytics drugs such as nebulised dornase alfa and hypertonic saline combined with regular chest physiotherapy and airway clearance techniques. ${ }^{20-23}$

Exocrine pancreatic insufficiency (PI) arises in CF due to the thick secretions that block the pancreatic ducts. This leads to decreased digestion and absorption of fats, protein and the fat-soluble vitamins A, D, E and $\mathrm{K} .{ }^{24} \mathrm{Up}$ to $85 \%$ of the people with CF have PI and require long-term pancreatic enzyme replacement therapy and replacement of fat soluble vitamins. ${ }^{2,25}$ Careful monitoring of growth and nutrition is therefore essential in PI patients. ${ }^{26,27}$ Accumulation of viscous material within the bowel can cause obstruction, presenting as meconium ileus in the neonatal period or leading to distal intestinal obstruction syndrome (DIOS) in older children and adults. ${ }^{24}$

In the liver, CFTR is expressed in the bile ducts, regulating the fluid and electrolyte content of the bile. A wide spectrum of liver disease may occur in people with $\mathrm{CF}$, from steatosis and neonatal cholestasis to more severe multilobular cirrhosis necessitating liver transplantation. ${ }^{28}$ 


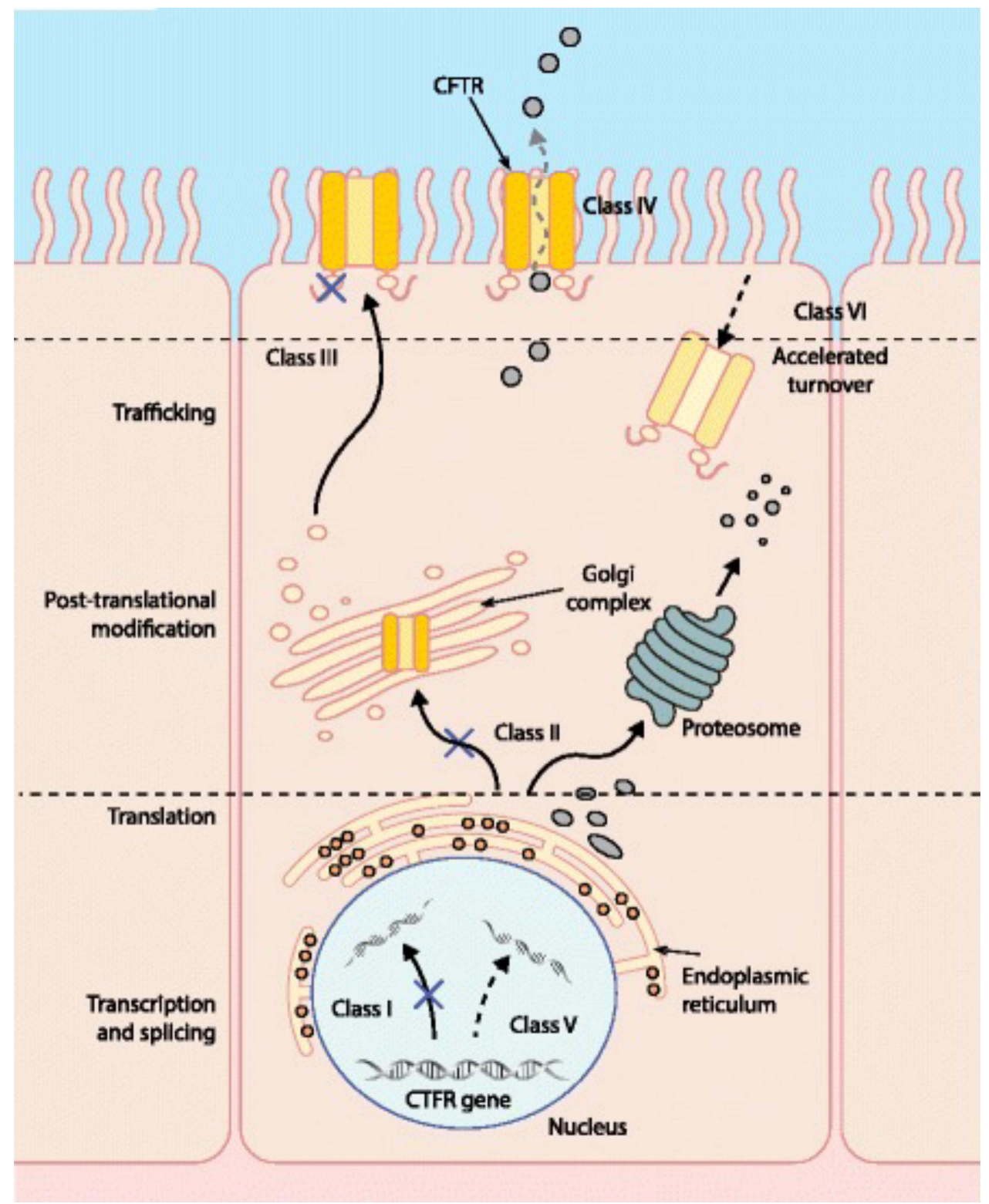

Figure 2 Schematic diagram of different CFTR mutation classes. Mutation classes I, II, V and VI result in an absence or reduced quantity of CFTR protein at the cell membrane, whereas mutation classes III and IV influence the function or activity of CFTR at the cell membrane. Potentiators increase the function of CFTR channels expressed at the apical surface of epithelial cells; for example, ivacaftor increases the probability of Gly55IAsp-CFTR channel opening. Correctors improve the intracellular processing and delivery of mutant CFTR protein, allowing more to reach the cell surface; for example, tezacaftor in Phe508del-CFTR. Adapted from Brodlie M, Haq IJ, Roberts K, Elborn JS. Targeted therapies to improve CFTR function in cystic fibrosis. Genome Med. Sep 24 2015;7:101. This article is distributed under the terms of the Creative Commons Attribution 4.0 International License (http://creativecommons.org/licenses/by/4.0/). ${ }^{87}$

CFRD affects around $40-50 \%$ of the adults with $\mathrm{CF}^{29}$ CFRD is caused by a relative insulin deficiency due to pancreas destruction and it shares some characteristics of both type I and type II diabetes with both islet cell loss and insulin resistance. $^{30}$

Fertility is also affected in CF. In the male reproductive tract, congenital absence of the vas deferens and azoospermia frequently leads to infertility; however, assisted fertility techniques are successful. ${ }^{31}$ Women have an anatomically normal reproductive tract, but cervical mucus abnormalities can lead to subfertility. ${ }^{2}$ 
Table 2 Definitions of CFTR Modulator Types

\begin{tabular}{|l|l|}
\hline Modulator Type & Summary of Action \\
\hline Potentiators & Restore or enhance channel gating and therefore chloride transport by CFTR at the cell membrane \\
\hline Correctors & Improve CFTR protein folding, processing and trafficking to the cell membrane \\
\hline Read-through agents & Supress premature stop codons in nonsense mutations to restore CFTR protein production \\
\hline Amplifiers & Stabilise CFTR mRNA to increase the amount of immature CFTR protein produced \\
\hline
\end{tabular}

\section{Targeting CFTR Function with Small-Molecule Drugs}

The ability to classify and understand the functional effects and molecular biology of common disease-causing CFTR variants has been pivotal in enabling the development of 'CFTR modulators' to ameliorate underlying defects and restore CFTR function. These include "potentiators" targeting specifically class III and IV variants and "correctors" used in combination with potentiators for class II variants (see Table 2). These drugs are now clinically available for the majority of people with CF and are the main focus of this article. Other pharmacological approaches include "read-through" agents for class I, "stabilisers" for class VI and "amplifiers" to increase translational efficacy across a range of variant classes, which are all also briefly summarised.

\section{Potentiator Therapies: Ivacaftor as an Example}

Ivacaftor (VX-770 or Kalydeco ${ }^{\mathrm{TM}}$, Vertex Pharmaceuticals) was the first CFTR potentiator approved for clinical use. It was initially licensed in Europe and the US for patients over 12 years with at least one copy of the gating G551D CFTR variant in 2012. It has since been approved for a variety of other disease-causing variants both as single therapy and in combination with other modulator drugs and for infants as young as 4 months of age with gating variants.

Ivacaftor was identified through high-throughput screening of over 200,000 compounds. ${ }^{32}$ In vitro studies in cell lines and primary human bronchial epithelial cell cultures (HBEs) showed that ivacaftor potentiated CFTR function, which improved chloride secretion and restored airway surface hydration and ciliary function in HBEs expressing G551D CFTR. $^{32}$ A definitive mechanism of action for ivacaftor has thus far remained elusive; however, there is evidence to suggest a unique phosphorylation-dependent mechanism increases channel open probability. ${ }^{33}$ In 2019, cryo-electron microscopy revealed an ivacaftor binding site in the transmembrane region of CFTR. ${ }^{34}$

The first Phase 3 randomised placebo controlled trial (RCT) to evaluate the efficacy of ivacaftor was performed in 161 patients $\geq 12$ years with at least one G551D CFTR variant over 48 weeks. ${ }^{35}$ Those treated with ivacaftor had a significant increase in percent predicted forced expiratory volume in 1 second $\left(\mathrm{ppFEV}_{1}\right)$ of 10.4 percentage points from baseline. Statistically and clinically significant benefits in quality of life, weight, reduced pulmonary exacerbations and sustained improvement in sweat chloride were also evident. A subsequent RCT of 52 participants aged 6 to 11 years with

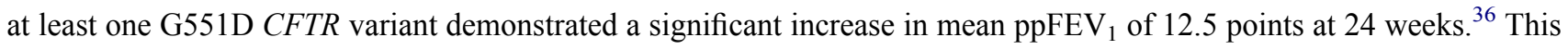
was sustained at 48 weeks with a 10-point increase in ppFEV1 along with improvements in weight, parent/carer reported quality of life and sweat chloride. ${ }^{36}$ Ivacaftor was later found to be efficacious in patients over 6 years and those aged $2-5$ years leading to extended approvals for use in these younger patients. ${ }^{37-39}$

Ivacaftor efficacy was also assessed for the residual function mutation, $\mathrm{R} 117 \mathrm{H}$ in patients aged 6 years and over. ${ }^{40}$ Although an absolute increase in $\mathrm{ppFEV}_{1}$ of 2.6 points was seen at 24 weeks, this was not statistically significant. However, significant improvements in sweat chloride and quality of life were observed. Notably, subgroup analysis showed that ppFEV1 significantly improved by 5 points in participants aged 18 years or older, but this was not apparent in those aged 6-11 years. An open-label extension study showed significant improvements in ppFEV1 of 5.5 from baseline in patients taking ivacaftor after a washout period. ${ }^{40}$

Ivacaftor is now approved by the US Food and Drug Administration (FDA) for patients aged over 4 months who are heterozygous for nine gating variants and R117H. Data from the 24-week open-label safety study (ARRIVAL) demonstrated 
a similar safety profile to that seen in older children and adults. ${ }^{41}$ In 2021, licensing for ivacaftor in the UK was extended to include a number of other variants of "varying clinical consequence" partially as a response to the COVID-19 pandemic. ${ }^{42}$

\section{Corrector and Potentiator Combination Therapies Lumacaftor/lvacaftor (Orkambi ${ }^{\mathrm{TM}}$ )}

Lumacaftor (VX-809, Vertex Pharmaceuticals), in combination with ivacaftor, was the first CFTR corrector to be licensed for clinical use by the FDA. It was identified via high throughput screening of small molecules aiming to increase chloride transport in cells expressing F508del CFTR. Initial in vitro studies were promising and demonstrated a fourfold increase in chloride transport. ${ }^{43}$ A subsequent Phase 2 clinical trial caused a modest reduction in sweat chloride, and although was not adequately powered to assess clinical outcomes, unfortunately did not live up to the promise in lung function outcomes as suggested by the in vitro data. ${ }^{44}$

Considering the combined effect of abnormal CFTR folding and conductance evident in F508del, the combination of both a corrector and potentiator therefore appeared a promising strategy to address both defects. Early clinical trials suggested that the combination of lumacaftor/ivacaftor improved both sweat chloride and lung function in F508del homozygous patients. ${ }^{45}$

In 2015, the results from two, phase 3, parallel group RCTs demonstrated a modest improvement in lung function in the ivacaftor/lumacaftor cohort. ${ }^{46}$ Absolute ppFEV1 increased by 2.4-4 points over the 24-week study period. A reduction in pulmonary exacerbations was also noted (30\% for $600 \mathrm{mg}$ once daily and $39 \%$ for $400 \mathrm{mg}$ twice daily). Adverse events reported more frequently in the treatment group were respiratory in nature, including most commonly chest tightness (10.8\%) and dyspnoea (14.9\%).

Subsequently, phase 3 studies were performed in a younger population. Lumacaftor/ivacaftor combination therapy in F508del homozygous patients aged between 6 and 11 years led to an improvement in absolute ppFEV1 of 2.4 points versus placebo and a significant reduction in sweat chloride $(-20.8 \mathrm{mmol} / \mathrm{l}) .{ }^{47}$ Given the relatively well-preserved lung function of this younger population lung clearance index values were measured alongside traditional spirometry, as a more sensitive marker of early lung damage. The absolute change in lung clearance index was $-1.09(-1.43$ to -0.75$)$ for treatment versus placebo. A similar rate of adverse events was reported when compared to trials in adult populations. A phase 3 study of lumacaftor/ivacaftor in F508del homozygous children aged between 2 and 5 years demonstrated comparable safety data and a significant reduction in sweat chloride. ${ }^{48}$

Lumacaftor/ivacaftor (Orkambi ${ }^{\mathrm{TM}}$ ) is currently licensed for use in the UK, Europe, the US and Australia for those who are over 2 years and F508del homozygous.

\section{Tezacaftor/lvacaftor (Symkevi ${ }^{\mathrm{TM}}$ )}

Tezacaftor is a corrector similar in structure to lumacaftor. It has comparable efficacy but importantly fewer reported adverse effects compared with lumacaftor/ivacaftor, which have led to high discontinuation rates. A phase 3 RCT published in 2017 evaluated the efficacy and safety of tezacaftor/ivacaftor combination therapy versus placebo in F508del homozygous patients aged 12 years and older. ${ }^{49}$ It reported an increase in absolute ppFEV of $_{1} .0$ points and a $35 \%$ reduction in pulmonary exacerbations in the treatment group. The rate of adverse events was similar in both treatment and placebo groups. Importantly, in contrast to lumacaftor/ivacaftor there was no increase in adverse respiratory events in the treatment group. A further trial investigating tezacaftor/ivacaftor in patients who had stopped treatment with lumacaftor/ivacaftor due to adverse respiratory events supported these findings. ${ }^{50}$ It demonstrated no increased rate of respiratory events with tezacaftor/ivacaftor compared to placebo and no patients in the trial discontinued treatment due to respiratory events.

Following on from successful trials in F508del homozygous patients an RCT investigated tezacaftor/ivacaftor in heterozygous patients with a single F508del mutation and a second mutation associated with residual CFTR function. ${ }^{51}$ This trial demonstrated a significant improvement in the absolute change in ppFEV1 in patients taking both ivacaftor monotherapy (4.7) and tezacaftor/ivacaftor (6.8) with a modest but significant difference favouring combination treatment. 
More recent Phase 3 trials investigating the effects of tezacaftor/ivacaftor in people heterozygous for F508del and either a minimal function or gating variant did not show significant improvements in lung function, despite a favourable safety profile in both studies. ${ }^{52,53}$

Tezacaftor/ivacaftor (Symkevi ${ }^{\mathrm{TM}}$ ) is currently licensed for use in homozygous F508del patients or heterozygous F508del patients with one residual function mutation. After further phase 3 trials confirmed safety and efficacy in a younger age group, both the FDA and subsequently the European Commission extended the licence to include children aged 6 years and older.

\section{Elexacaftor/Tezacaftor/lvacaftor "Triple Therapy" (Kaftrio ${ }^{\mathrm{TM}}$ or Trikafta ${ }^{\mathrm{TM}}$ )}

Whilst double combination therapy had significantly advanced treatment of people with F508del, improvements in sweat chloride and pulmonary function were relatively modest. Furthermore, around $30 \%$ of individuals heterozygous for F508del and a minimally functioning mutation did not respond to an available modulator treatment. ${ }^{52}$ Sustained efforts to improve processing and trafficking of the F508del CFTR protein led to the addition of a second CFTR corrector. The rationale for this was based on the observation of synergistic effects of correctors with different proposed mechanisms/ sites of action. ${ }^{54}$ Four agents were identified that showed promise in early preclinical and clinical trials. From these, the VX-445 and VX-659 compounds were selected for further investigation based on efficacy and side effect profiles.

In vitro investigation showed that when used alongside tezacaftor/ivacaftor, both compounds significantly improved F508del CFTR processing, trafficking and chloride transport when compared to tezacaftor/ivacaftor alone. In patients, efficacy was demonstrated in both F508del homozygous and heterozygous individuals, including those with a minimally functioning second mutation, as described below. ${ }^{55,56}$

Phase 2 clinical trials were constructed with two separate arms. The first arm, in homozygous patients, allowed comparison of triple therapy with the next best current treatment of tezacaftor/ivacaftor alone. The second arm included heterozygous patients with a minimally functioning second variant. Since no current CFTR modulator therapy was recommended for this group of patients, triple therapy was compared with placebo. In homozygous patients both compounds demonstrated a significant improvement in $\mathrm{ppFEV}_{1}$ (VX-445: 11.0 and VX-659: 9.7 point increases). Crucially, a marked improvement in $\mathrm{ppFEV}_{1}$ was demonstrated in the heterozygous study group (VX-445: 13.8 and VX-659: 13.3 point increases). Reported adverse effects for both compounds were largely mild or moderate. ${ }^{55,56}$

Results of follow-on phase 3 clinical trials exceeded expectations. In heterozygous individuals treated with VX-445 triple therapy, there was an improvement in ppFEV ${ }_{1}$ of 13.8 points at week 4 and 14.3 points by week 24 when compared with placebo. ${ }^{57}$ On average, pulmonary exacerbation rates were $63 \%$ lower, sweat chloride had reduced by $41 \mathrm{mmol} / \mathrm{L}$ and body mass index increased by $1.04 \mathrm{~kg} / \mathrm{m}^{2}$ over the trial period.

Results were also impressive in the homozygous population with an increase in $\mathrm{ppFEV}_{1}$ of 10 points and a reduction in sweat chloride of $45.1 \mathrm{mmol} / 1$ at 4 weeks when compared to dual tezacaftor/ivacaftor therapy. ${ }^{58}$ The study period for this arm was reduced as previous trials in this population had confirmed that improvements seen at 4 weeks were sustained at 24 and 48 weeks. Safety data for both trials were reassuring with predominantly mild or moderate events and similar levels of events reported in both placebo and treatment groups. A greater number of participants in the treatment arm had raised liver transaminases (10.9\% vs $4 \%)$.

VX-659 progressed to further phase 3 trials with encouraging outcomes. ${ }^{59,60}$ However, based on safety and efficacy data VX-445 was selected as a preferable compound to take forward and is marketed by Vertex Pharmaceuticals as elexacaftor. ${ }^{61}$

Based on the above data, the FDA approved the triple combination of elexacaftor/tezacaftor/ivacaftor for use in patients aged $\geq 12$ years with at least one F508del mutation in October 2019, just 2 months after application. At the time of writing, January 2022, the FDA, the UK Medicines and Healthcare products Regulatory Agency and the European Medicines Agency have now expanded approval in children aged 6 to 11 years with at least one F508del mutation or CFTR disease-causing variants that show in vitro responses to treatment. ${ }^{62}$

In 2021, a phase 3 RCT of patients aged 12 years and over with a combination of either F508del and a gating mutation or F508del and a residual function mutation found that elexacaftor/tezacaftor/ivacaftor increased $\mathrm{ppFEV}_{1}$ by 3.5 
points in patients already receiving ivacaftor or tezacaftor/ivacaftor at the start of the trial, with added significant improvements in sweat chloride. ${ }^{63}$

\section{"Real-World" Experience of Modulator Therapies}

The clinical trials outlined above have been vital in enabling access to modulator therapies. However, such trials represent a relatively stable population selected from strict inclusion and exclusion criteria. Data derived from "realworld" post-licensing observational studies may in fact better represent patient populations who would have otherwise been excluded from clinical trials.

As the first licensed CFTR modulator, the majority of published real-world data relates to ivacaftor treatment. One systematic review of 57 observational studies, including the large GOAL study in the US, investigated the impact of ivacaftor in adult and paediatric populations up to a 5-year period and demonstrated that in the majority of cases, the mean absolute change in ppFEV ${ }_{1}$ from baseline increased by 5-10 percentage points. ${ }^{64} \mathrm{~A}$ recent longitudinal, singlecentre, study of adults with G551D showed that acute benefits in ppFEV ${ }_{1}$ were not sustained and at 5 years ppFEV had $_{1}$ declined to pre-treatment levels in those individuals. ${ }^{65}$ This is in keeping with the findings from the multicentre prospective GOAL study of G551D patients over 5.5 years, which also showed that adults demonstrated greater sustained benefits in $\mathrm{ppFEV}_{1}$ than children. ${ }^{66}$ It is possible that ivacaftor treatment has long-term implications on preservation rather than absolute increase in lung function, as demonstrated in the larger systematic review, where lung function was in fact better preserved in ivacaftor-treated cohorts for up to 5 years compared with cohorts that had not received any modulator treatment at all. ${ }^{64}$ Notably, GOAL demonstrated significant decreases in pulmonary exacerbations and infection with Pseudomonas aeruginosa and Aspergillus spp. Improvements in quality of life have been found to be sustained at 5.5 years. ${ }^{66}$

At present, the implications of modulators on the overall burden of care for people with CF is unclear; however, it is anticipated that this will be alleviated in the future. One aspect that remains to be determined is the impact on pancreatic exocrine function and need for PERT. A paediatric study of 28 children has shown a significant reduction in PERT consumption after 1 year of ivacaftor treatment, but further data is currently lacking and it has only been published in abstract form. ${ }^{67}$ Long-term studies in the UK and US of other CF-related co-morbidities have shown that although CFRD prevalence increases in both ivacaftor-treated and untreated cohorts at up to 5 years from baseline, the magnitude of this increase was lower in ivacaftor-treated cohorts. ${ }^{64}$ Individuals treated with ivacaftor have also shown improvements in BMI, lower rates of bone/joint disease, depression and hepatobiliary complications, with reduced frequency of organ transplantation in those with severe disease compared to untreated cohorts both in the UK and US. ${ }^{64}$ Notably, ivacaftor treatment in those with advanced CF disease in the UK and Ireland, who were part of a compassionate use programme, was associated after 3 years of treatment with a lower rate of death. ${ }^{68}$

The impact of lumacaftor/ivacaftor treatment was evaluated in a recent large French multi-centre study and showed that although treatment was associated with ongoing improvements in $\mathrm{pFEV}_{1}$, nutritional status and requirements for intravenous antibiotics, there were no significant improvements in HbAlc or levels of fat-soluble vitamins. Eighteen percent of patients discontinued treatment due to predominantly respiratory adverse events including chest tightness and bronchospasm, which were more apparent in those with severe disease (ppFEV $1<40 \%) .{ }^{69}$

Information relating to the real-world impact of elexacaftor/tezacaftor/ivacaftor is currently limited due to its relatively recent approval. Importantly, there is some evidence of clinical benefit in people with advanced lung disease, a cohort of patients who have not been included in clinical trials yet there is an urgent need for effective treatments. ${ }^{70}$ A case series of patients with advanced lung disease receiving elexacaftor/tezacaftor/ivacaftor as part of a managed access programme in Ireland showed significant improvements in $\mathrm{ppFEV}_{1}, \mathrm{BMI}$ and CFRD. ${ }^{71}$ Careful consideration is required of clinically significant drug interactions, especially following transplantation, for example, with calcineurin inhibitors or azole antifungals. ${ }^{70}$

Post-licensing studies also suggest that there is a higher frequency of reported adverse events with lumacaftor/ ivacaftor compared with other modulators, which are more pronounced in those with severe lung disease. These are predominantly respiratory in nature and include symptoms of dyspnoea and chest tightness, often occurring at treatment initiation but have led to discontinuation in up to $30 \%$ of the patients. ${ }^{69,70,72}$ These effects have not been seen with 
ivacaftor alone, or with other modulator combinations, raising the suggestion that they may be specific to lumacaftor. Several strategies have been used to minimise these effects including reducing the starting dose and co-administration with an inhaled bronchodilator in patients with severe disease. ${ }^{70}$

Elevated blood pressure has been reported in clinical trials for lumacaftor/ivacaftor and elexacaftor/tezacaftor/ ivacaftor. ${ }^{72}$ Other reported adverse events include liver enzyme derangement, which is typically transient, headache and fatigue specifically reported with lumacaftor/ivacaftor and rash with all available modulator combinations, but these effects are seldom associated with cessation of treatment. ${ }^{72}$ Rarer events associated with all modulators include elevated creatinine kinase levels, which have been reported in both clinical trials and population-based studies and have shown an association with increased physical activity. ${ }^{72}$ Cataracts have also rarely been reported with ivacaftor monotherapy and lumacaftor/ivacaftor treatments and form the basis of guidance around regular eye screening at baseline and annually for those receiving treatment. ${ }^{72}$

There have been reported negative associations of CFTR modulators on mental health, but the exact mechanisms for this remain unclear. Visual hallucinations, migraine and sleep disturbance have been reported in a small number of individuals receiving tezacaftor/ivacaftor and there are reported cases of depression and anxiety with lumacaftor/ ivacaftor. ${ }^{72}$ A recent case series of six patients receiving elexacaftor/tezacaftor/ivacaftor reported short-term memory loss, insomnia, "brain fog", and word-finding difficulty from 1 month of starting treatment. ${ }^{73}$ Symptoms resolved by stopping treatment, dose reduction or swapping the morning and evening doses where insomnia was a significant factor. Further work is required to determine the long-term effects of modulator therapies on mental health, particularly at the time where the mental health of many people has suffered during the COVID-19 pandemic.

Clearly, as further experience with modulator therapies increases, there is a significant need to monitor and report adverse events that may arise in the future. This highlights the importance of working closely with pharmacy colleagues within the CF multi-disciplinary team to consider strategies to reduce adverse events and minimise drug interactions. Furthermore, whilst significant improvements in BMI and nutrition are very much welcomed in patients with severe disease, the role of the specialist dietician in CF care is evolving to manage the emerging challenges of excessive weight gain and obesity particularly in children and those with more stable disease.

\section{Therapies in Development}

Read-Through Agents

Despite the welcome successful development of CFTR modulator therapy for many people with CF, different approaches are required to target other classes of CFTR variants. Specifically, class I variants caused by premature termination codons (PTCs), result in a shortened dysfunctional CFTR protein and a severe clinical phenotype. ${ }^{74}$ Therapeutic approaches to these variants include read-through agents, which promote the production of full-length CFTR. Ataluren is one such compound that despite showing early promise did not show benefit in larger clinical trials. ${ }^{74-76}$ Clinical investigation of other read-through agents, including ELX-02 (Eloxx Pharmaceuticals), is currently underway, and the results are awaited.

\section{Amplifiers}

Amplifiers are compounds that stabilise CFTR mRNA and increase the amount of immature CFTR protein produced. ${ }^{77,78}$ They were identified through high throughput screening and are of relevance to class $\mathrm{V}$ variants or, in combination with correctors and potentiators, all other variants except those where no mRNA is generated. Beneficial effects have been demonstrated in preclinical studies and are yet to be investigated in clinical trials.

\section{Other Potentiators and Correctors}

Other modulators in the development pipeline include the potentiator galicaftor (ABBV-2222) and corrector navocaftor (ABBV-3067) developed by AbbVie. ABBV-2222 alone did not show significant improvements in lung function in phase 2 clinical trials but was well tolerated and is currently under investigation in combination with ABBV-3067. ${ }^{79,80}$ ABBV- 
119 is another potentiator, and further phase 2 trials are underway of the triple combination of ABBV-119/galicaftor/ navocaftor. $^{81}$

\section{Genetic Therapies}

Clearly, since CF is a genetic condition caused by variants in the CFTR gene, strategies to replace or repair this genetic defect are attractive. ${ }^{82}$ This potentially curative gene therapy approach has become available to people with spinal muscular atrophy recently. ${ }^{83}$ However, despite significant efforts, gene therapy in CF has been challenged by difficulties in suppressing the host immune response and maintaining gene expression. ${ }^{84,85}$ Nevertheless, efforts are ongoing and alternative approaches including the application of alternative adeno-associated and lentiviral vectors and methods to repair defective CFTR mRNA are under investigation. ${ }^{86,87}$ Gene editing involving CRISPR/Cas-9 technologies have potential to incorporate a functional CFTR gene into a patient's cells and hold promise for a curative therapy. ${ }^{82,87}$ Ongoing research will undoubtedly involve exploration of how such treatments can be effectively delivered to anatomically challenging organs such as the lung and the airway epithelium. ${ }^{85,86}$ Alternatively, it may be possible in the future to edit epithelial progenitor cells ex vivo and then return and engraft the corrected cells. ${ }^{85}$ It has been recognised through the development of single-cell transcriptomics that individual subtypes of epithelial cells exist in the airway and have variable expression of CFTR. For example, ionocytes are present in small numbers but highly express CFTR ${ }^{88}$ Further understanding in this area may inform which types of epithelial cells should be targeted for the greatest clinical benefit. ${ }^{85}$

\section{Closing Discussion}

The development of CFTR modulator therapies has been a major success in CF therapeutics. At the time of the discovery of the CFTR gene in 1989, average life expectancy was significantly reduced for people with CF. Almost 40 years later, the majority of people with CF are now eligible for treatment with CFTR modulators. These therapies have been highly effective in clinical trials. The long-term benefits are yet to be determined, however, observational post-licensing studies do suggest sustained "real-world" benefits. ${ }^{89,90}$ Implications for overall treatment burden are also starting to be explored. For example, the SIMPLIFY and CFSTORM (https://www.cfstorm.org.uk/) studies are investigating the withdrawal of nebulised mucolytic treatment. ${ }^{91}$

Variability in modulator response is recognised in individuals with CF, despite sharing the same CFTR genotype. Furthermore, large-scale RCTs are simply not feasible for many rare disease-causing variants. A number of ex vivo models have been developed to allow testing of an individual's response to specific modulators, thus allowing appropriate targeted therapy or "theranostics". ${ }^{92}$ Primary human nasal epithelial cells (HNEs) can be obtained from nasal mucosal brushings. ${ }^{93}$ Conditionally reprogrammed expansion of HNEs can increase the number of cultures available for ex vivo investigation of ion transport and response to modulators. Organoid cultures have also been developed from intestinal biopsies and nasal and bronchial cells isolated from individuals with $\mathrm{CF}$, which provide a 3D model where forskolininduced swelling (FIS) provides a higher throughput assessment of response to modulators. ${ }^{92,94,95}$

These models have been employed in larger scale programmes designed to assess the effectiveness of modulators, particularly for individuals with rarer CFTR disease-causing variants, namely in the European HIT-CF (https://www.hitcf. org/) and the Canadian CFIT (https://lab.research.sickkids.ca/cfit/) programmes. Patient-derived models are the "gold standard" tool for drug discovery in CF, but other cell line-based assays have been utilised to advance research. In particular, Fisher Rat Thyroid (FRT) cells expressing CFTR variants have been employed by the FDA to investigate and approve modulator therapy for individuals with rare variants. Induced pluripotent stem cells (iPSCs) can also be differentiated into multiple cell types and used to investigate modulator responses. ${ }^{96}$

Despite the uptake of these models, it is important to recognise their limitations, which include specific variations in culture techniques between research laboratories and variability between cell samples derived from the same individuals. ${ }^{92,97}$ Persistence of epigenetic variation in cultured cells caused by exposure to specific environmental factors may also lead to variations in in vitro responses. 


\section{Conclusion}

The rapid translation of CFTR modulators from their identification using in vitro models through to large RCTs and their subsequent widespread clinical use in many countries is a major success story of biomedical research and precision medicine. In particular, ivacaftor and elexacaftor/tezacaftor/ivacaftor appear to be a disease-modifying transformative development in $\mathrm{CF}$ care that generates improvements in quality of life and lung health that are clinically highly significant. It is important to note that results of long-term follow-up are not yet available, but clinical benefits appear to be sustained.

Crucially, around $10 \%$ of the people with CF do not have a modulator option and for this cohort, ongoing research to develop therapeuticsfor example, in gene editing and other gene-based therapies, remain vital for this "lost $10 \%$ ". Equally, the high cost of CFTR modulators has been another major issue that has limited access to these drugs in some healthcare systems worldwide. ${ }^{98}$ Equitable access must be a priority and costs should be offset against savings in longterm healthcare as people with CF remain healthier.

\section{Acknowledgments}

We are grateful for support to fund CF research in our group from the Medical Research Council, Wellcome and Cystic Fibrosis Trust.

\section{Disclosure}

MB received investigator-led research grants from Pfizer and Roche Diagnostics; speaker fees paid to Newcastle University from Novartis, Roche Diagnostics and TEVA. Travel expenses to educational meetings Boehringer Ingelheim and Vertex Pharmaceuticals. Miss Simone Soars reports grants from Cystic Fibrosis Trust. The authors report no other conflicts of interest in this work.

\section{References}

1. Riordan J, Rommens J, Kerem B, et al. Identification of the cystic fibrosis gene: cloning and characterization of complementary DNA. Science. 1989;245(4922):1066-1073. doi:10.1126/science.2475911

2. Shteinberg M, Haq IJ, Polineni D, Davies JC. Cystic fibrosis. Lancet. 2021;397(10290):2195-2211. doi:10.1016/S0140-6736(20)32542-3

3. Castellani C, Massie J, Sontag M, Southern KW. Newborn screening for cystic fibrosis. Lancet Respir Med. 2016;4(8):653-661. doi:10.1016/ S2213-2600(16)00053-9

4. Trust C. UK cystic fibrosis registry annual data report 2019; 2020. Available from: https://www.cysticfibrosis.org.uk/sites/default/files/2020-12/ 2019\%20Registry\%20Annual\%20Data\%20report_Sep\%202020.pdf. Accessed January 26, 2022.

5. Cystic Fibrosis Trust. Cystic Fibrosis Trust: Standards for the Clinical Care of Children and Adults with Cystic Fibrosis in the UK. Second ed. Cystic Fibrosis Trust; 2021.

6. Zielenski J, Rozmahel R, Bozon D, et al. Genomic DNA sequence of the cystic fibrosis transmembrane conductance regulator (CFTR) gene. Genomics. 1991;10(1):214-228. doi:10.1016/0888-7543(91)90503-7

7. Sheppard DN, Welsh MJ. Structure and function of the CFTR chloride channel. Physiol Rev. 1999;79(1):S23-S45. doi:10.1152/physrev.1999.79.1.S23

8. Hwang T-C, Sheppard DN. Gating of the CFTR Cl- channel by ATP-driven nucleotide-binding domain dimerisation. J Physiol. 2009;587 (10):2151-2161. doi:10.1113/jphysiol.2009.171595

9. Vergani P, Lockless SW, Nairn AC, Gadsby DC. CFTR channel opening by ATP-driven tight dimerization of its nucleotide-binding domains. Nature. 2005;433:876. doi:10.1038/nature03313

10. Schloesser M, Arleth S, Lenz U, Bertele RM, Reiss J. A cystic fibrosis patient with the nonsense mutation G542X and the splice site mutation 1717-1. J Med Genet. 1991;28(12):878. doi:10.1136/jmg.28.12.878

11. Sheppard DN, Rich DP, Ostedgaard LS, Gregory RJ, Smith AE, Welsh MJ. Mutations in CFTR associated with mild-disease-form CI- channels with altered pore properties. Nature. 1993;362(6416):160-164. doi:10.1038/362160a0

12. Beck S, Penque D, Garcia S, et al. Cystic fibrosis patients with the 3272-26A $\rightarrow$ G mutation have mild disease, leaky alternative mRNA splicing, and CFTR protein at the cell membrane. Hum Mutat. 1999;14(2):133-144. doi:10.1002/(SICI)1098-1004(1999)14:2<133::AID-HUMU5>3.0. $\mathrm{CO} ; 2-\mathrm{T}$

13. Ramalho AS, Lewandowska MA, Farinha CM, et al. Deletion of CFTR translation start site reveals functional isoforms of the protein in CF patients. Cell Physiol Biochem. 2009;24(5-6):335-346. doi:10.1159/000257426

14. Mishra A, Greaves R, Smith K, et al. Diagnosis of cystic fibrosis by sweat testing: age-specific reference intervals. J Pediatr. 2008;153(6):758763.e1. doi:10.1016/j.jpeds.2008.04.067

15. Haq IJ, Gray MA, Garnett JP, Ward C, Brodlie M. Airway surface liquid homeostasis in cystic fibrosis: pathophysiology and therapeutic targets. Thorax. 2016;71(3):284-287. doi:10.1136/thoraxjnl-2015-207588

16. Morrison CB, Markovetz MR, Ehre C. Mucus, mucins, and cystic fibrosis. Pediatr Pulmonol. 2019;54(Suppl 3):S84-S96. doi:10.1002/ ppul.24530 
17. Pezzulo AA, Tang XX, Hoegger MJ, et al. Reduced airway surface $\mathrm{pH}$ impairs bacterial killing in the porcine cystic fibrosis lung. Nature. 2012;487(7405):109-113. doi:10.1038/nature11130

18. Keown K, Brown R, Doherty DF, et al. Airway inflammation and host responses in the era of CFTR modulators. Int J Mol Sci. 2020;21 (17):6379. doi:10.3390/ijms21176379

19. Coutinho HDM, Falcão-Silva VS, Gonçalves GF. Pulmonary bacterial pathogens in cystic fibrosis patients and antibiotic therapy: a tool for the health workers. Int Arch Med. 2008;1(1):24. doi:10.1186/1755-7682-1-24

20. Jones AP, Wallis C. Dornase alfa for cystic fibrosis. Cochrane Database Syst Rev. 2010;(3). doi:10.1002/14651858.CD001127.pub2

21. Fuchs HJ, Borowitz DS, Christiansen DH, et al. Effect of aerosolized recombinant human DNase on exacerbations of respiratory symptoms and on pulmonary function in patients with cystic fibrosis. N Engl J Med. 1994;331(10):637-642. doi:10.1056/NEJM199409083311003

22. Elkins MR, Robinson M, Rose BR, et al. A controlled trial of long-term inhaled hypertonic saline in patients with cystic fibrosis. $N$ Engl $J$ Med. 2006;354(3):229-240. doi:10.1056/NEJMoa043900

23. Main E, Prasad A, van der Schans CP. Conventional chest physiotherapy compared to other airway clearance techniques for cystic fibrosis. Cochrane Database Syst Rev. 2005;(1). doi:10.1002/14651858.CD002011.pub2

24. Somaraju URR, Solis-Moya A. Pancreatic enzyme replacement therapy for people with cystic fibrosis. Cochrane Database Syst Rev. 2020;(8). doi:10.1002/14651858.CD008227.pub4

25. Stern RC, Eisenberg JD, Wagener JS, et al. A comparison of the efficacy and tolerance of pancrelipase and placebo in the treatment of steatorrhea in cystic fibrosis patients with clinical exocrine pancreatic insufficiency. Am J Gastroenterol. 2000;95(8):1932-1938. doi:10.1016/ S0002-9270(00)01036-4

26. Nutritional basics. Available from: https://www.cff.org/Life-With-CF/Daily-Life/Fitness-and-Nutrition/Nutrition/Getting-Your-Nutrients /Nutritional-Basics/. Accessed February 22, 2021.

27. Hollander FM, de Roos NM, Dopheide J, Hoekstra T, van Berkhout FT. Self-reported use of vitamins and other nutritional supplements in adult patients with cystic fibrosis. Is daily practice in concordance with recommendations? Int J Vitamin Nutr Res. 2010;80(6):408-415. doi:10.1024/ 0300-9831/a000025

28. Boëlle P-Y, Debray D, Guillot L, Clement A, Corvol H; on behalf of the French CFMGSI. Cystic fibrosis liver disease: outcomes and risk factors in a large cohort of French patients. Hepatology. 2019;69(4):1648-1656. doi:10.1002/hep.30148

29. Moran A, Dunitz J, Nathan B, Saeed A, Holme B, Thomas W. Cystic fibrosis-related diabetes: current trends in prevalence, incidence, and mortality. Diabetes Care. 2009;32(9):1626. doi:10.2337/dc09-0586

30. Marshall BC, Butler SM, Stoddard M, Moran AM, Liou TG, Morgan WJ. Epidemiology of cystic fibrosis-related diabetes. J Pediatr. 2005 ; 146 (5):681-687. doi:10.1016/j.jpeds.2004.12.039

31. Chillón M, Casals T, Mercier B, et al. Mutations in the cystic fibrosis gene in patients with congenital absence of the vas deferens. $N$ Engl $J$ Med. 1995;332(22):1475-1480. doi:10.1056/NEJM199506013322204

32. Van Goor F, Hadida S, Grootenhuis PDJ, et al. Rescue of CF airway epithelial cell function in vitro by a CFTR potentiator, VX-770. Proc Am Thorac Soc. 2009;106(44):18825-18830. doi:10.1073/pnas.0904709106

33. Eckford PD, Li C, Ramjeesingh M, Bear CE. Cystic fibrosis transmembrane conductance regulator (CFTR) potentiator VX-770 (ivacaftor) opens the defective channel gate of mutant CFTR in a phosphorylation-dependent but ATP-independent manner. J Biol Chem. 2012;287 (44):36639-36649. doi:10.1074/jbc.M112.393637

34. Liu F, Zhang Z, Levit A, et al. Structural identification of a hotspot on CFTR for potentiation. Science. 2019;364(6446):1184-1188. doi:10.1126/science.aaw7611

35. Ramsey BW, Davies J, McElvaney NG, et al. A CFTR potentiator in patients with cystic fibrosis and the G551D mutation. $N$ Engl $J$ Med. 2011;365(18):1663-1672. doi:10.1056/NEJMoa1105185

36. Davies JC, Wainwright CE, Canny GJ, et al. Efficacy and safety of ivacaftor in patients aged 6 to 11 years with cystic fibrosis with a G551D mutation. Am J Respir Crit Care Med. 2013;187(11):1219-1225. doi:10.1164/rccm.201201-0153OC

37. De Boeck K, Munck A, Walker S, et al. Efficacy and safety of ivacaftor in patients with cystic fibrosis and a non-G551D gating mutation. $J$ Cyst Fibros. 2014;13(6):674-680. doi:10.1016/j.jcf.2014.09.005

38. Rosenfeld M, Cunningham S, Harris WT, et al. An open-label extension study of ivacaftor in children with CF and a CFTR gating mutation initiating treatment at age 2-5 years (KLIMB). J Cystic Fibrosis. 2019;18(6):838-843. doi:10.1016/j.jcf.2019.03.009

39. Davies JC, Cunningham S, Harris WT, et al. Safety, pharmacokinetics, and pharmacodynamics of ivacaftor in patients aged 2-5 years with cystic fibrosis and a CFTR gating mutation (KIWI): an open-label, single-arm study. Lancet Respir Med. 2016;4(2):107-115. doi:10.1016/ S2213-2600(15)00545-7

40. Moss RB, Flume PA, Elborn JS, et al. Efficacy and safety of ivacaftor in patients with cystic fibrosis who have an Arg117His-CFTR mutation: a double-blind, randomised controlled trial. Lancet Respir Med. 2015;3(7):524-533. doi:10.1016/S2213-2600(15)00201-5

41. Davies JC, Wainwright CE, Sawicki GS, et al. Ivacaftor in infants aged 4 to $<12$ months with cystic fibrosis and a gating mutation. results of a two-part phase 3 clinical trial. Am J Respir Crit Care Med. 2021;203(5):585-593. doi:10.1164/rccm.202008-3177OC

42. England NHS. Clinical Commissioning Urgent Policy Statement Ivacaftor and tezacaftor/ivacaftor for cystic fibrosis: "off-label" use in patients with named rarer mutations; 2021. Available from: https://www.england.nhs.uk/wp-content/uploads/2020/08/Urgent-policy-statement-CFTR-off -label-rarer-mutations.pdf. Accessed January 26, 2022.

43. Van Goor F, Hadida S, Grootenhuis PD, et al. Correction of the F508del-CFTR protein processing defect in vitro by the investigational drug VX-809. Proc Natl Acad Sci U S A. 2011;108(46):18843-18848. doi:10.1073/pnas.1105787108

44. Clancy J, Rowe SM, Accurso FJ, et al. Results of a phase IIa study of VX-809, an investigational CFTR corrector compound, in subjects with cystic fibrosis homozygous for the F508del-CFTR mutation. Thorax. 2012;67(1):12-18. doi:10.1136/thoraxjnl-2011-200393

45. Boyle MP, Bell SC, Konstan MW, et al. A CFTR corrector (lumacaftor) and a CFTR potentiator (ivacaftor) for treatment of patients with cystic fibrosis who have a phe508del CFTR mutation: a phase 2 randomised controlled trial. Lancet Respir Med. 2014;2(7):527-538. doi:10.1016/ S2213-2600(14)70132-8

46. Wainwright CE, Elborn JS, Ramsey BW, et al. Lumacaftor-ivacaftor in patients with cystic fibrosis homozygous for Phe508del CFTR. $N$ Engl $J$ Med. 2015;373(3):220-231. doi:10.1056/NEJMoa1409547 
47. Ratjen F, Hug C, Marigowda G, et al. Efficacy and safety of lumacaftor and ivacaftor in patients aged 6-11 years with cystic fibrosis homozygous for F508del-CFTR: a randomised, placebo-controlled phase 3 trial. Lancet Respir Med. 2017;5(7):557-567. doi:10.1016/S2213-2600(17)30215-1

48. McNamara JJ, McColley SA, Marigowda G, et al. Safety, pharmacokinetics, and pharmacodynamics of lumacaftor and ivacaftor combination therapy in children aged 2-5 years with cystic fibrosis homozygous for F508del-CFTR: an open-label phase 3 study. Lancet Respir Med. 2019;7 (4):325-335. doi:10.1016/S2213-2600(18)30460-0

49. Taylor-Cousar JL, Munck A, McKone EF, et al. Tezacaftor-ivacaftor in patients with cystic fibrosis homozygous for Phe508del. $N$ Engl J Med. 2017;377(21):2013-2023. doi:10.1056/NEJMoa1709846

50. Schwarz C, Sutharsan S, Epaud R, et al. Tezacaftor/ivacaftor in people with cystic fibrosis who stopped lumacaftor/ivacaftor due to respiratory adverse events. J Cyst Fibros. 2021;20(2):228-233. doi:10.1016/j.jcf.2020.06.001

51. Rowe SM, Daines C, Ringshausen FC, et al. Tezacaftor-ivacaftor in residual-function heterozygotes with cystic fibrosis. $N$ Engl $J$ Med. 2017;377(21):2024-2035. doi:10.1056/NEJMoa1709847

52. Munck A, Kerem E, Ellemunter H, et al. Tezacaftor/ivacaftor in people with cystic fibrosis heterozygous for minimal function CFTR mutations. J Cyst Fibros. 2020;19(6):962-968. doi:10.1016/j.jcf.2020.04.015

53. McKone EF, DiMango EA, Sutharsan S, et al. A phase 3, randomized, double-blind, parallel-group study to evaluate tezacaftor/ivacaftor in people with cystic fibrosis heterozygous for F508del-CFTR and a gating mutation. J Cyst Fibros. 2021;20(2):234-242. doi:10.1016/j. jcf.2020.11.003

54. Okiyoneda T, Veit G, Dekkers JF, et al. Mechanism-based corrector combination restores DeltaF508-CFTR folding and function. Nat Chem Biol. 2013;9(7):444-454. doi:10.1038/nchembio.1253

55. Davies JC, Moskowitz SM, Brown C, et al. VX-659-tezacaftor-ivacaftor in patients with cystic fibrosis and one or two Phe508del alleles. N Engl J Med. 2018;379(17):1599-1611. doi:10.1056/NEJMoa1807119

56. Keating D, Marigowda G, Burr L, et al. VX-445-tezacaftor-ivacaftor in patients with cystic fibrosis and one or two Phe508del alleles. $N$ Engl $J$ Med. 2018;379(17):1612-1620. doi:10.1056/NEJMoa1807120

57. Middleton PG, Mall MA, Dřevínek P, et al. Elexacaftor-tezacaftor-ivacaftor for cystic fibrosis with a single Phe508del allele. $N$ Engl $J$ Med. 2019;381(19):1809-1819. doi:10.1056/NEJMoa1908639

58. Heijerman HGM, McKone EF, Downey DG, et al. Efficacy and safety of the elexacaftor plus tezacaftor plus ivacaftor combination regimen in people with cystic fibrosis homozygous for the F508del mutation: a double-blind, randomised, phase 3 trial. Lancet. 2019;394 (10212):1940-1948. doi:10.1016/S0140-6736(19)32597-8

59. Clinicaltrials.gov. A phase 3 study of VX-659 combination therapy in subjects with cystic fibrosis heterozygous for the F508del mutation and a minimal function mutation (F/MF); 2021. Available from: https:/clinicaltrials.gov/ct2/show/results/NCT03447249. Accessed January 26, 2022.

60. Vertex. Two phase 3 studies of the triple combination of VX-659, tezacaftor and ivacaftor met primary endpoint of improvement in lung function (ppFEV1) in people with cystic fibrosis; 2022. Available from: https://investors.vrtx.com/news-releases/news-release-details/two-phase -3-studies-triple-combination-vx-659-tezacaftor-and. Accessed January 14, 2022.

61. Vertex. Vertex selects triple combination regimen of VX-445, tezacaftor and ivacaftor to submit for global regulatory approvals in cystic fibrosis; 2022. Available from: https://investors.vrtx.com/news-releases/news-release-details/vertex-selects-triple-combination-regimen-vx-445tezacaftor-and. Accessed January 14, 2022.

62. England NHS. 'Miracle' cystic fibrosis treatment for children on the NHS; 2022. Available from: https://www.england.nhs.uk/2022/01/miraclecystic-fibrosis-treatment-for-children-on-the-nhs/. Accessed January 14, 2022.

63. Barry PJ, Mall MA, Álvarez A, et al. Triple therapy for cystic fibrosis Phe508del-gating and -residual function genotypes. $N$ Engl $J$ Med. 2021;385(9):815-825. doi:10.1056/NEJMoa2100665

64. Duckers J, Lesher B, Thorat T, et al. Real-world outcomes of ivacaftor treatment in people with cystic fibrosis: a systematic review. J Clin Med. 2021;10(7):1527. doi:10.3390/jcm10071527

65. Mitchell RM, Jones AM, Stocking K, Foden P, Barry PJ. Longitudinal effects of ivacaftor and medicine possession ratio in people with the Gly551Asp mutation: a 5-year study. Thorax. 2021;76(9):874-879. doi:10.1136/thoraxjnl-2020-215556

66. Guimbellot JS, Baines A, Paynter A, et al. Long term clinical effectiveness of ivacaftor in people with the G551D CFTR mutation. $J$ Cyst Fibros. 2021;20(2):213-219. doi:10.1016/j.jcf.2020.11.008

67. Emery J, Mullane D, Chroinin MN. GP284 The effects of ivacaftor on pancreatic function in paediatric patients with cystic fibrosis gating mutations. Arch Dis Child. 2019;104(Suppl 3):A149-A150. doi:10.1136/archdischild-2019-epa.343

68. Barry PJP, Simmonds BJ, Bicknell NJ, et al. Ivacaftor decreases mortality in G551D patients with severe lung disease. Pediatr Pulmonol. 2015;50:275-276.

69. Burgel PR, Munck A, Durieu I, et al. Real-life safety and effectiveness of lumacaftor-ivacaftor in patients with cystic fibrosis. Am J Respir Crit Care Med. 2020;201(2):188-197. doi:10.1164/rccm.201906-1227OC

70. Benden C, Schwarz C. CFTR modulator therapy and its impact on lung transplantation in cystic fibrosis. Pulm Ther. 2021;7(2):377-393. doi:10.1007/s41030-021-00170-9

71. O'Shea KM, O'Carroll OM, Carroll C, et al. Efficacy of elexacaftor/tezacaftor/ivacaftor in patients with cystic fibrosis and advanced lung disease. Eur Respir J. 2021;57(2). doi:10.1183/13993003.03079-2020

72. Dagenais RVE, Su VCH, Quon BS. Real-world safety of CFTR modulators in the treatment of cystic fibrosis: a systematic review. J Clin Med. 2020;10(1):23. doi:10.3390/jcm10010023

73. Heo S, Young DC, Safirstein J, et al. Mental status changes during elexacaftor/tezacaftor/ivacaftor therapy. J Cyst Fibros. 2021. doi:10.1016/j. jef.2021.10.002

74. Zainal Abidin N, Haq IJ, Gardner AI, Brodlie M. Ataluren in cystic fibrosis: development, clinical studies and where are we now? Expert Opin Pharmacother. 2017;18(13):1363-1371. doi:10.1080/14656566.2017.1359255

75. Kerem E, Konstan MW, De Boeck K, et al. A randomized placebo-controlled trial of ataluren for the treatment of nonsense mutation cystic fibrosis. Lancet Respir Med. 2014;2(7):539-547. doi:10.1016/S2213-2600(14)70100-6 
76. Konstan MW, VanDevanter DR, Rowe SM, et al. Efficacy and safety of ataluren in patients with nonsense-mutation cystic fibrosis not receiving chronic inhaled aminoglycosides: the international, randomized, double-blind, placebo-controlled Ataluren Confirmatory Trial in Cystic Fibrosis (ACT CF). J Cystic Fibrosis. 2020;19:1873-5010.

77. Dukovski D, Villella A, Bastos C, et al. Amplifiers co-translationally enhance CFTR biosynthesis via PCBP1-mediated regulation of CFTR mRNA. J Cyst Fibros. 2020;19(5):733-741. doi:10.1016/j.jcf.2020.02.006

78. Giuliano KA, Wachi S, Drew L, et al. Use of a high-throughput phenotypic screening strategy to identify amplifiers, a novel pharmacological class of small molecules that exhibit functional synergy with potentiators and correctors. SLAS Discov. 2018;23(2):111-121. doi:10.1177/ 2472555217729790

79. Cystic Fibrosis Foundation. Drug development pipeline; 2021. Available from: https://apps.cff.org/trials/pipeline/details/10145/ABBV-2222formerly-GLPG2222. Accessed January 26, 2022.

80. Bell SC, Barry PJ, De Boeck K, et al. CFTR activity is enhanced by the novel corrector GLPG2222, given with and without ivacaftor in two randomized trials. J Cyst Fibros. 2019;18(5):700-707. doi:10.1016/j.jcf.2019.04.014

81. Clinicaltrials.gov. Study to evaluate adverse events and change in disease activity with oral capsules of galicaftor/navocaftor/ABBV-119 combination therapy in adult participants with cystic fibrosis; 2021. Available from: https://clinicaltrials.gov/ct2/show/NCT04853368. Accessed January 26, 2022.

82. Egan ME. Emerging technologies for cystic fibrosis transmembrane conductance regulator restoration in all people with CF. Pediatr Pulmonol. 2021;56(Suppl 1):S32-S39. doi:10.1002/ppul.24965

83. Goemans N. Gene therapy for spinal muscular atrophy: hope and caution. Lancet Neurol. 2021;20(4):251-252. doi:10.1016/S1474-4422(21)00071-5

84. Alton E, Armstrong DK, Ashby D, et al. Repeated nebulisation of non-viral CFTR gene therapy in patients with cystic fibrosis: a randomised, double-blind, placebo-controlled, phase 2b trial. Lancet Respir Med. 2015;3(9):684-691. doi:10.1016/S2213-2600(15)00245-3

85. Christopher Boyd A, Guo S, Huang L, et al. New approaches to genetic therapies for cystic fibrosis. J Cyst Fibros. 2020;19(Suppl 1):S54-S59. doi:10.1016/j.jcf.2019.12.012

86. Ensinck M, Mottais A, Detry C, Leal T, Carlon MS. On the corner of models and cure: gene editing in cystic fibrosis. Front Pharmacol. 2021;12:662110. doi:10.3389/fphar.2021.662110

87. Brodlie M, Haq IJ, Roberts K, Elborn JS. Targeted therapies to improve CFTR function in cystic fibrosis. Genome Med. $2015 ; 7: 101$. doi:10.1186/s13073-015-0223-6

88. Montoro DT, Haber AL, Biton M, et al. A revised airway epithelial hierarchy includes CFTR-expressing ionocytes. Nature. 2018;560 (7718):319-324. doi:10.1038/s41586-018-0393-7

89. Bessonova L, Volkova N, Higgins M, et al. Data from the US and UK cystic fibrosis registries support disease modification by CFTR modulation with ivacaftor. Thorax. 2018;73(8):731-740. doi:10.1136/thoraxjnl-2017-210394

90. Volkova N, Moy K, Evans J, et al. Disease progression in patients with cystic fibrosis treated with ivacaftor: data from national US and UK registries. J Cyst Fibros. 2020;19(1):68-79. doi:10.1016/j.jcf.2019.05.015

91. Mayer-Hamblett N, Nichols DP, Odem-Davis K, et al. Evaluating the impact of stopping chronic therapies after modulator drug therapy in cystic fibrosis: the SIMPLIFY clinical trial study design. Ann Am Thorac Soc. 2021;18(8):1397-1405. doi:10.1513/AnnalsATS.202010-1336SD

92. Amaral MD, de Boeck K, Amaral M, et al. Theranostics by testing CFTR modulators in patient-derived materials: the current status and a proposal for subjects with rare CFTR mutations. J Cyst Fibros. 2019;18(5):685-692. doi:10.1016/j.jcf.2019.06.010

93. Haq IJ, Althaus M, Gardner AI, et al. Clinical and molecular characterization of the R751L-CFTR mutation. Am J Physiol Lung Cell Mol Physiol. 2021;320(2):L288-L300. doi:10.1152/ajplung.00137.2020

94. Dekkers JF, Berkers G, Kruisselbrink E, et al. Characterizing responses to CFTR-modulating drugs using rectal organoids derived from subjects with cystic fibrosis. Sci Transl Med. 2016;8:344ra84-344ra84. doi:10.1126/scitranslmed.aad8278

95. Sette G, Lo Cicero S, Blaconà G, et al. Theratyping cystic fibrosis in vitro in ALI culture and organoid models generated from patient-derived nasal epithelial conditionally reprogrammed stem cells. Eur Respir J. 2021;58:2100908. doi:10.1183/13993003.00908-2021

96. Merkert S, Bednarski C, Göhring G, Cathomen T, Martin U. Generation of a gene-corrected isogenic control iPSC line from cystic fibrosis patient-specific iPSCs homozygous for p.Phe508del mutation mediated by TALENs and ssODN. Stem Cell Res. 2017;23:95-97. doi:10.1016/j. scr.2017.07.010

97. Matthes E, Goepp J, Martini C, et al. Variable responses to CFTR correctors in vitro: estimating the design effect in precision medicine. Front Pharmacol. 2018;9(1490). doi:10.3389/fphar.2018.01490

98. Lopes-Pacheco M. CFTR modulators: the changing face of cystic fibrosis in the era of precision medicine. Front Pharmacol. $2019 ; 10: 1662$. doi: $10.3389 /$ fphar.2019.01662

99. Pinto MC, Silva IAL, Figueira MF, Amaral MD, Lopes-Pacheco M. Pharmacological modulation of ion channels for the treatment of cystic fibrosis. J Exp Pharmacol. 2021;13:693-723. doi:10.2147/JEP.S255377

100. Liu F, Zhang Z, Csanady L, Gadsby DC, Chen J. Molecular structure of the human CFTR ion channel. Cell. 2017;169(1):85-95 e8. doi:10.1016/j.cell.2017.02.024

101. Zhang Z, Liu F, Chen J. Molecular structure of the ATP-bound, phosphorylated human CFTR. Proc Natl Acad Sci U S A. 2018;115 (50):12757-12762. doi:10.1073/pnas.1815287115

Pharmacogenomics and Personalized Medicine

Dovepress

\section{Publish your work in this journal}

Pharmacogenomics and Personalized Medicine is an international, peer-reviewed, open access journal characterizing the influence of genotype on pharmacology leading to the development of personalized treatment programs and individualized drug selection for improved safety, efficacy and sustainability. This journal is indexed on the American Chemical Society's Chemical Abstracts Service (CAS). The manuscript management system is completely online and includes a very quick and fair peer-review system, which is all easy to use. Visit http://www. dovepress.com/testimonials.php to read real quotes from published authors.

Submit your manuscript here: https://www.dovepress.com/pharmacogenomics-and-personalized-medicine-journa: 